\title{
A DECOMPOSITION OF BOUNDED SCALARLY MEASURABLE FUNCTIONS TAKING THEIR RANGES IN DUAL BANACH SPACES
}

\author{
ELIZABETH M. BATOR
}

(Communicated by Jerry J. Uhl, Jr.)

\begin{abstract}
A decomposition of scalarly measurable functions taking their range in the dual of a Banach space into a Pettis integrable part and a weak* scalarly null part is introduced and analyzed.
\end{abstract}

1. Introduction. It is shown in [11] that if $X$ is a separable Banach space, $K$ is a compact Hausdorff space, and $f: K \rightarrow X^{*}$ is a bounded universally scalarly measurable function, then $f$ is universally Pettis integrable. In order to prove this, they first show the following:

LEMMA. Let $(\Omega, \Sigma, \mu)$ be a finite measure space and let $X$ be a separable Banach space. If $f: \Omega \rightarrow X^{*}$ is a bounded scalarly measurable function such that for every $\varepsilon>0$, there exists $E \in \Sigma$ such that $\mu(\Omega \backslash E)<\varepsilon$ and $\left\{\langle f, x\rangle_{E}:\|x\| \leq 1\right\}$ is weakly precompact in $L^{\infty}(\mu)$, then $f$ is $\mu$-Pettis integrable.

They also point out that the separability hypothesis in both of these cannot be removed. In particular there is an example due to Phillips (see Example B in the next section) of a function $f:[0,1] \rightarrow l^{\infty}[0,1]$ such that $f$ is scalarly Borel measurable but not Lebesgue Pettis integrable.

In [8] Musial shows that if $X$ is a separable Banach space then the following statements are equivalent:

(i) $X$ does not contain any isomorphic copy of $l^{1}$.

(ii) $X^{*}$ has the weak Radon Nikodym property.

(iii) If $(\Omega, \Sigma, \mu)$ is a complete measure space and $f: \Omega \rightarrow X^{*}$ is bounded and weak $^{*}$ scalarly measurable, then $f$ is Pettis integrable.

(iv) If $(\Omega, \Sigma, \mu)$ is a complete measure space and $f: \Omega \rightarrow X^{*}$ is bounded and weak $^{*}$ scalarly measurable, then $f$ is scalarly measurable.

Janika was able to extend the equivalence of statements (i) and (ii) for the case $X$ not necessarily separable in [7]. However, when we remove the separability hypothesis, statements (i) and (ii) are not equivalent to statements (iii) and (iv) as seen in Example $\mathrm{C}$ in the next section.

The purpose of this paper is to extend the above-mentioned results to the case $X$ not necessarily separable by a suitable weakening of the conclusion.

Received by the editors March 11, 1986 and, in revised form, September 22, 1986. Presented January 11, 1986 at the annual meeting of the AMS in New Orleans, Louisiana.

1980 Mathematics Subject Classification (1985 Revision). Primary 28B05, 46G10.

Key words and phrases. Banach space, Pettis integral, scalarly measurable.

Partially supported by Faculty Research Grant from North Texas State University. 
Let us introduce some definitions and preliminary facts. Let $(\Omega, \Sigma, \mu)$ be a finite measure space and $X$ be a Banach space. A function $f: \Omega \rightarrow X^{*}$ is scalarly measurable if $x^{* *} \circ f$ is measurable for every $x^{* *} \in X^{* *}$, and $f$ is weak scalarly measurable if $x \circ f$ is measurable for every $x \in X$. The function $f$ is $\mu$-Pettis integrable if for every $E \in \Sigma$, there exists $x_{E}^{*} \in X^{*}$ such that

$$
x^{* *}\left(x_{E}^{*}\right)=\int_{E} x^{* *} \circ f d \mu \text { for every } x^{* *} \in X^{* *} .
$$

In this case, $x_{E}^{*}=\mathrm{P}-\int_{E} f d \mu$.

If $K$ is a compact Hausdorff space, then $f: K \rightarrow X^{*}$ is universally scalarly measurable if $f$ is scalarly measurable with respect to all Radon measures on $K$. A weak* compact subset $K$ of $X^{*}$ is called a Pettis set if the canonical injection $K \rightarrow X^{*}$ is scalarly measurable for each Radon measure on $\left(K\right.$, weak $\left.{ }^{*}\right)$.

A subset $K$ of a Banach space $X$ is called weakly precompact if every bounded sequence has a weakly Cauchy subsequence. Rosenthal's theorem [10] states that a bounded subset $K$ of a Banach space $X$ is weakly precompact if and only if $K$ does not contain an isomorphic copy of the basis of $l^{1}$.

A Banach space $X$ has the weak Radon Nikodym property (WRNP) if for every finite measure space $(\Omega, \Sigma, \mu)$, every operator $T: L^{1}(\mu) \rightarrow X^{*}$ is Pettis representable, i.e. there exists a Pettis integrable function $f$ such that $T(g)=\mathrm{P}-\int f g d \mu$.

2. Weak* scalarly null functions. Let $X$ be a Banach space and let $(\Omega, \Sigma, \mu)$ be a finite measure space. We define a function $f: \Omega \rightarrow X^{*}$ to be $\mu$-weak* scalarly null if $x \circ f=0$ a.e.- $[\mu]$ for every $x \in X$.

Proposition 1. Let $X$ be a separable Banach space. Then $f$ is $\mu$-weak* scalarly null if and only if $f=0$ a.e.- $[\mu]$.

ProOF. Let $\left(x_{j}\right)_{j=1}^{\infty}$ be dense in $X$. Let $N_{j}$ be $\mu$-null sets such that $x_{j} \circ f(t)=0$ if $t \in \Omega \backslash N_{j}$. Clearly $f(t)=0$ if $t \in \Omega \backslash\left(\bigcup_{j=1}^{\infty} N_{j}\right)$.

Consequently any interesting example of a weak ${ }^{*}$ scalarly null function will occur only if the function takes its range in a nonseparable space.

Examples. In each of the following examples, $\mu$ is Lebesgue measure on $[0,1]$ and $\Sigma$ is the Lebesgue measurable subsets of $[0,1]$.

Example A. Let $X=l^{2}[0,1]$. Define $f:[0,1] \rightarrow l^{2}[0,1]=X^{*}$ by

$$
f(s)(t)= \begin{cases}1 & \text { if } s=t, \\ 0 & \text { if } s \neq t .\end{cases}
$$

If $x \in l^{2}[0,1]$ then $x \circ f$ is countably nonzero since $x$ has countable support. Therefore, $f$ is $\mu$-weak ${ }^{*}$ scalarly null. It is also easy to see that $f$ is $\mu$-Pettis integrable and that $\mathrm{P}-\int_{E} f d \mu=0$ for every $E \in \Sigma$, even though $f$ is not equal to zero a.e.- $[\mu]$.

EXAMPLE B (PhILliPs). Let $X=l^{1}[0,1]$. Let $B$ be the subset of $[0,1] \times[0,1]$ constructed by Sierpinski having the following properties:

(i) for every $t_{0} \in[0,1],\left\{s:\left(s, t_{0}\right) \in B\right\}$ is countable.

(ii) for every $s_{0} \in[0,1],\left\{t:\left(s_{0}, t\right) \notin B\right\}$ is countable. Define $f:[0,1] \rightarrow l^{\infty}[0,1]$ by $f(s)(t)=\chi_{B}(s, t)$. It is shown in [5] that $f$ is bounded and scalarly Borel measurable but not $\mu$-Pettis integrable. If $x \in l^{1}[0,1]$, it is clear that $x \circ f$ is countably nonzero and $f$ is $\mu$-weak* scalarly null. 
EXAMPLE C. Let $f:[0,1] \rightarrow l^{1}[0,1]$ be given by $f(t)=e_{t}$. If $x \in c_{0}[0,1]$, then again $x \circ f$ is countably nonzero and $f$ is $\mu$-weak ${ }^{*}$ scalarly null. However, if $A$ is any subset of $[0,1]$, then $\chi_{A} \in l^{\infty}[0,1]$ and $\chi_{A} \circ f=\chi_{A}$. Hence $f$ is not scalarly measurable.

The above examples show that a nontrivial weak* scalarly null function may be Pettis integrable, scalarly measurable but not Pettis integrable, or even not scalarly measurable.

PROPOSITION 2. Let $X$ be a Banach space and $(\Omega, \Sigma, \mu)$ be a finite measure space. If $f: \Omega \rightarrow X^{*}$ is bounded and scalarly measurable then the following are equivalent:

(i) There exists a $\mu$-Pettis integrable function $g$ and a $\mu$-weak* scalarly null function $h$ such that $f=g+h$.

(ii) There exists a $\mu$-Pettis integrable function $g$ such that for every $x^{* *} \in X^{* *}$, $T_{f}^{* *}\left(x^{* *}\right)=x^{* *} \circ g$ in $L^{1}(\mu)$.

(iii) For every $\varepsilon>0$, there exists $A \in \Sigma$ and a Pettis integrable function $g$ such that $\mu(\Omega \backslash A)<\varepsilon$ and $(x \circ f) \chi_{A}=x \circ g$ a.e. $[\mu]$ for every $x \in X$.

ProOF. (i) $\rightarrow$ (ii) If $f=g+h$ and $h$ is weak ${ }^{*}$ scalarly null, then $x \circ f=x \circ g$ in $L^{1}(\mu)$ for every $x \in X$. Since $g$ is Pettis integrable it is clear that $T_{g}^{* *}\left(x^{* *}\right)=x^{* *} \circ g$ in $L^{1}(\mu)$ for every $x^{* *} \in X^{* *}$. Hence we have that for every $x^{* *} \in X^{* *}$,

$$
T_{f}^{* *}\left(x^{* *}\right)=T_{g}^{* *}\left(x^{* *}\right)=x^{* *} \circ g \text { in } L^{1}(\mu) .
$$

(ii) $\rightarrow$ (iii) Clear.

(iii) $\rightarrow$ (i) Use a standard exhaustion argument as in Lemma III.2.4 in [3] to get a Pettis integrable function $g$ such that $x \circ f=x \circ g$ a.e.- $[\mu]$ for every $x \in X$. Clearly $h=f-g$ is $\mu$-weak* scalarly null.

We say a bounded scalarly measurable function is $\mu$-Pettis decomposable if any and hence all of the above statements are true. An immediate consequence of Proposition 1 is that if $X$ is a separable Banach space then a bounded scalarly measurable function $f: \Omega \rightarrow X^{*}$ is Pettis decomposable if and only if $f$ is Pettis integrable.

3. Decomposition and the RS-property. In [13], Talagrand defines a weak* measurable function $f: \Omega \rightarrow X^{*}$ to have the $R S$-property if the Radon image measure $\nu=\mu \circ f^{-1}$ is such that for every $n$ there is a Pettis set $K_{n}$ such that $\nu\left(\Omega \backslash K_{n}\right) \leq 1 / 2^{n}$. He then shows that if $f$ is weak ${ }^{*}$ scalarly bounded, then $f$ has the RS-property if and only if for every $\varepsilon>0$ there exists $E \in \Sigma$ with $\mu(\Omega \backslash E)<\varepsilon$ such that the set $\left\{\langle f, x\rangle \chi_{E}:\|x\| \leq 1\right\}$ is weakly precompact. Therefore the Lemma of Riddle, Saab, and Uhl mentioned in the introduction can be stated as follows: If $X$ is a separable Banach space and $f: \Omega \rightarrow X^{*}$ is bounded, scalarly measurable and has the RS-property, then $f$ is Pettis integrable. We are now able to extend this result.

THEOREM 3. Let $(\Omega, \Sigma, \mu)$ be a finite measure space and $X$ a Banach space. If $f: \Omega \rightarrow X^{*}$ is a bounded scalarly measurable function such that $f$ has the $R S$ property, the $f$ is $\mu$-Pettis decomposable.

PROOF. By Theorem 7-3-15 of [13], if $f$ has the RS-property, then there exists a Pettis integrable function $g: \Omega \rightarrow X^{*}$ such that $x \circ f=x \circ g$ in $L^{1}(\mu)$ for every 
$x$ in the unit ball of $X$. Consequently, by part (iii) of Proposition $2, f$ is $\mu$-Pettis decomposable.

In Proposition 7-3-16 of [13], Talagrand exhibits a Pettis integrable function $\varphi:[0,1] \rightarrow l^{\infty}$ that does not have the RS-property. Obviously $\varphi$ is Pettis decomposable. Hence it is clear that decomposability is strictly weaker than the RS-property.

Talagrand raises the following question: If $K$ is a compact Hausdorff space and $f: K \rightarrow X^{*}$ is universally scalarly measurable, must $f$ have the RS-property for every Radon measure $\mu$ on $K$ ? It is also natural to ask the following:

QUESTION. If $K$ is a compact Hausdorff space, $X$ is a Banach space, and $f: K \rightarrow$ $X^{*}$ is bounded and universally scalarly measurable, must $f$ be $\mu$-decomposable for every Radon measure $\mu$ ?

In [10], Riddle and Saab show that the answer to Talagrand's question is yes if $f$ is universally Lusin measurable, and in fact such an $f$ must be Pettis integrable.

4. Pettis decomposition and the weak Radon Nikodym property. We begin this section with the following observation.

LEMMA 4. Let $(\Omega, \Sigma, \mu)$ be a perfect measure space and $f: \Omega \rightarrow X^{*}$ be a bounded weak $^{*}$ scalarly measurable function. If $f=g+h$, where $g$ is scalarly measurable and $h$ is weak* scalarly null, then the operator $T_{f}: X \rightarrow L^{1}(\mu)$, defined by $T_{f}(x)=x \circ f$ for every $x \in X$, is compact.

PROOF. Since $h$ is weak* scalarly null, $T_{f}(x)=T_{g}(x)$ in $L^{1}(\mu)$ for every $x \in X$. However, since $g$ is scalarly measurable, the operator $T_{g}$ is compact by Proposition 3 of $[\mathbf{1}]$.

We are now able to prove the following extension of Musial's result.

THEOREM 5. If $X$ is a Banach space, then the following are equivalent:

(i) $X$ does not contain an isomorphic copy of $l^{1}$.

(ii) $X^{*}$ has the WRNP.

(iii) If $(\Omega, \Sigma, \mu)$ is a complete measure space and $f: \Omega \rightarrow X^{*}$ is bounded and weak* $^{*}$ scalarly measurable, then $f$ is Pettis decomposable.

(iv) If $(\Omega, \Sigma, \mu)$ is a complete measure space and $f: \Omega \rightarrow X^{*}$ is bounded and weak $^{*}$ scalarly measurable, then $f=g+h$, where $g$ is scalarly measurable and $h$ is weak* scalarly null.

Proof. Janika proved the equivalence of (i) and (ii) in [7]. The fact that (ii) implies (iii) is given in Theorem 2 of [8]. We have only left to show (iv) implies (i).

It is shown in [2, Theorem 3.5] that if $X$ is a Banach space that contains $l^{1}$, then there exists a bounded weak ${ }^{*}$ Lebesgue measurable function $f:[0,1] \rightarrow X^{*}$ such that the operator $T_{f}: X \rightarrow L^{1}[0,1]$ is not compact. Hence by Lemma 4 , this function cannot satisfy the conclusion of statement (iv).

ADDENDUM. The question in $\S 3$ has been affirmitively answered by the author in "Pettis decomposition for universally scalarly measurable functions".

\section{REFERENCES}

1. E. M. Bator, The Pettis Integral and the equality of the norms of the Dunford integral and the weak* integral, Proc. Amer. Math. Soc. 95 (1985), 265-270.

2. E. M. Bator, P. W. Lewis, and D. Race, Some connections between Pettis integration and operator theory, Rocky Mountain Math. J. 17 (1987), 683-695. 
3. J. Diestel and J. J. Uhl, Jr., Vector measures, Math. Surveys, no. 15, Amer. Math. Soc., Providence, R.I., 1977.

4. N. Dunford and J. T. Schwartz, Linear operators, Part I, Interscience, New York, 1958.

5. R. F. Geitz, Pettis integration, Proc. Amer. Math. Soc. 82 (1981), 81-86.

6. __ Geometry and the Pettis integral, Trans. Amer. Math. Soc. 269 (1982), 535-548.

7. L. Janika, Some measure-theoretic characterizations of Banach spaces not containing ${ }^{1}{ }^{1}$, Bull. Acad. Polon. Sci. 27 (1979), 561-565.

8. K. Musial, The weak Radon-Nikodym property in Banach spaces, Studia. Math. 641 (1978), 151-174.

9. B. J. Pettis, On integration in vector spaces, Trans. Amer. Math. Soc. 44 (1938), 277-304.

10. L. H. Riddle and E. Saab, On functions that are universally Pettis integrable, Illinois J. Math. 29 (1985), 509-531.

11. L. H. Riddle, E. Saab, and J. J. Uhl, Jr., Sets with the weak Radon-Nikodym property in dual Banach spaces, Indiana Univ. Math. J. 32 (1983), 527-540.

12. H. P. Rosenthal, A characterization of Banach spaces containing $l^{1}$, Proc. Nat. Acad. Sci. U.S.A. 71 (1974), 2411-2413.

13. M. Talagrand, Pettis integral and measure theory, Mem. Amer. Math. Soc., vol. 51, no. 307, 1984.

Department of Mathematics, North Texas State University, Denton, Texas 76203 